\section{Multiple ligation of the proximal greater saphenous vein in the CHIVA treatment of primary varicose veins}

\author{
Roberto Delfrate, ${ }^{1}$ Massimo Bricchi, ${ }^{1}$ \\ Claude Franceschi, ${ }^{2}$ Matteo Goldoni ${ }^{3}$ \\ 'Surgery Unit, Figlie di San Camillo \\ Hospital, Cremona, Italy; ${ }^{2}$ Angiology \\ Consultant, Saint Joseph Hospital, Paris, \\ France; ${ }^{3}$ Department of Consulting of \\ Clinical Medicine, Nephrology and Health \\ Sciences, University of Parma, Italy
}

\section{Abstract}

Saphenous femoral disconnection is the key point of most surgical techniques in the treatment of primary varicose vein surgery. The aim of this study is to compare and analyze different techniques for conservative saphenousfemoral ligation or disconnection. These techniques can be to perform mini invasive open surgery and are suitable for implementation of the conservative hemodynamic correction of venous insufficiency (CHIVA) method. The aim was to present the follow-up by retrospective analysis of three different ligation-disconnection techniques of the proximal great saphenous vein (GSV) according to the CHIVA method at the GSV end, i.e. between the very end of the GSV and the first arch tributary, according to the CHIVA method. The first thecnique consisted of a surgical division (crossotomy). The other two consisted of triple superposed ligation with No. 2 non-absorbable braided coated suture without division labeled TSFL (triple saphenous flush ligation) and No. 0 polypropylenene ligation TPL (triple polypropylene ligation). The difference between TSFL and TPL was in the thickness and type of material of the thread, though both were non-absorbable. The follow up of 56 TPL procedures, 61 crossotomy procedures, and 82 TSFL procedures was analysed. The follow-up consisted of checking the sapheno-femoral junction occlusion with Duplex color ultra sound. The incidence rates of neovascularization (new vessels in the ligation or surgical disconnection site with saphenous-femoral reflux during the Valsalva maneuver) were: $4.9 \%$ for the crossotomy group, $6.1 \%$ for the TSFL group and $37.5 \%$ for the TPL group. The data analysed show satisfactory results with both crossotomy and TSFL. Crossotomy has proven to be an effective technique for performing saphenous-femoral disconnection, but TSFL could also be a reliable, safe and low-cost varicose mini-invasive surgery in outpatients. TPL appeared to be less reliable.

\section{Introduction}

The sapheno-femoral junction (SFJ) is a key point for the venous drainage of the lower limb from the foot up to the hip and gluteus, the lower abdominal wall and lower genital tract. Moreover, the disconnection of the incompetent SFJ is a fundamental procedure to most open superficial venous surgery. ${ }^{1-5}$ Unfortunately, most varicose recurrences are due to SFJ neovascularization (recurrences) observed in $25 \%$ to $94 \%$ of recurrent varicose veins. ${ }^{6}$ Conservative saphenous-femoral disconnection is a very common surgical practice according to the conservative hemodynamic correction of venous insufficiency (CHIVA) method. ${ }^{7-12}$ For more than a decade we have tested a technique that requires a division of the SFJ and others that require only peculiar ligatures without division of the incompetent saphenous femoral tract. The simplicity of the procedure, the safety of the maneuver, and the effectiveness of the treatment have been our goals.

\section{Materials and Methods}

SFJ disconnections and ligations were performed using three different techniques in patients affected by SFJ reflux through the terminal and also subterminal valves responsible for clinical disorders. All the varicose patients were assessed by Duplex ultra sound (US), where the SFJ reflux was checked with the Valsalva, squeezing and Paranà maneuvers with doppler sample on the femoral side of the terminal valve, and SFJ were skin marked using the Duplex US Scan probe (12 $\mathrm{MHz}$ probe). The techniques were performed in outpatients, under local anesthesia and by the same surgeon. The surgeon and other coauthors did the follow-up checkup using the same color doppler ultrasound equipment: in particular, neorevascularization was detected at the disconnection-ligation site using the color fuction during the Valsalva maneuver. The Valsalva maneuver was performed by having the patients to blow into a straw that was closed at one end: Cremona maneuver. ${ }^{13}$ In all the cases the Valsalva maneuver was performed after properly emptying of the deep venous system through the performance of a Parana maneuver with the aim to prevent false negatives caused by the presence of a full deep venous system. Parana is a gravitational test performed on a patient standing in front of the examiner who pushes the patient's back off balance, either backward (posterior Paranà maneuver) or forward (anterior Paranà maneuver). In order to maintain balance there is an isometric reactive contraction of the
Correspondence: Roberto Delfrate, Figlie di San Camillo Hospital, via Fabio Filzi 56, 26100 Cremona, Italy.

Tel.: +39.0372.421111 - Mobile: +39.334 .9089110 . E-mail: roberto.delfrate@icloud.com

Key words: saphenous-femoral disconnection, saphenous-femoral junction, neovascularization recurrences, primary varicose vein surgery.

Received for publication: 11 September 2013.

Revision received: 11 February 2014.

Accepted for publication: 6 March 2014.

This work is licensed under a Creative Commons Attribution 3.0 License (by-nc 3.0).

CCopyright R. Delfrate et al., 2014

Licensee PAGEPress, Italy

Veins and Lymphatics 2014; 3:1919

doi:10.4081/vl.2014.1919

muscular groups that activates venous circulation by emptying the deep venous system. All the SFJs were studied in order to rule out both a Valsalva of pelvic origin and one deriving from a lateral crural perforator.

\section{Surgical procedure}

The saphenous-femoral disconnection-ligation was performed between the SFJ and the first arch tributary, preserving both the arch tributaries and the great saphenous vein (GSV) trunk (Figure 1), while the refluxing tributaries were divided at their trunk connection. A titanium clip (10 mm long and $1 \mathrm{~mm}$ thick) was placed flush with the femoral vein in all cases of the the three groups in order to prevent the presence of a residual saphenous stump. The GSV ligation was performed with a No. 2 non-absorbable braided coated suture in the triple saphenous flush ligation (TSFL) group, and with No. 0 polypropylenene ligation in triple polypropylene ligation (TPL) group. The SFJ is exposed thanks to a previous suspension on silicone loops of the superficial epigastric vein, the pudendal vein and the great saphenous vein. The first GSV ligation is performed in a layer close to tributary outflow into the SFJ, the second one as close as possible to the femoral vein, and the third one in an intermediate position. The disconnection is called crossotomy when it consists of a division. It is called selective ligature when no interrution is performed. In case of crossotomy the fossa ovalis was closed with a polypropylene suture.

\section{Subjects and interventions}

The number of interventions and patients during follow-up visits are reported in Table 1.

The follow-up period was longer for crossotomy than the other two groups; in particular, in the TPL group the follow-up was always $\leq 12$ 
months. Crossotomy is the technique that was adopted and has been commonly used since 2003, while the TSFL experience began in 2007. Since 2007 the number of crossotomy procedures has therefore been reduced and this is the reason why crossotomy procedures usually require a longer follow-up.

\section{Statistical analysis}

Normally distributed variables were reported as mean; otherwise, as median (interquartile range). Differences among groups for continuous variables were assessed by means of ANOVA followed by Tukey's post-hoc test or the Kruskal-Wallis test followed by the Bonferroni post-hoc test. Differences in categorical variables were assessed by means of the chisquare test using the Bonferroni correction. Survival was considered as follows: i) the event was neovascularization for the crossotomy group, considered the control group; ii) the event was asymptomatic or symptomatic recanalization during the follow-up period. Kaplan-Meyer survival curve was calculated for the survival data followed by log-rank test with the Bonferroni correction which was calculated for the survival data to assess the differences among groups. An adjusted model for age and gender was further calculated using the Cox regression. All the models were repeated with and without bilateral surgery, when in the first case unilateral surgery; all the surgeries were considered as independent measures. $\mathrm{P}=0.05$ was the significance value. All the statistical tests were performed with SPSS 20 (IBM Corp., Chicago, IL, USA).

\section{Results}

Four hundred nine TSFL procedures where performed from January 2007 to January 2011. From January to May 2011, 82 procedures were controlled. The follow-up checks at six months and longer were only possible in 71 patients because some of the patients didn't want to undergo an examination, saying on the phone, that they were satisfied with the operation outcome and didn't feel that a follow-up was necessary. Five recurrences due to recanalization of the ligatures with saphenous-femoral reflux were detected at the procedure site in three patients (two bilateral, one unilateral). The reflux during the Valsalva test was limited to the SFJ in one case, it reached the GSV right below the preterminal valve in one case, in another case one it reached the proximal third of the thigh, and in another two cases was at mid-thigh level. Both patients who had had a bilateral procedure showed a reflux starting eight months after the operation and the follow-up was at 35 and 33 months for the male and at 31 to 26 months for the female. One patient who had had a unilateral procedure with saphenous-femoral reflux recurrence detected at 14 months after surgery didn't accept any further follow-up because he was satisfied with the clinical result. Whereas the two other patients were satisfied with the operation despite both showing a bilateral saphenous-femoral reflux at the thigh: in one case at the preterminal valve, and in three cases at the middle of the thigh. Total recurrences amounted to $5 / 82=6.1 \%$ of all cases treated. The four cases of reflux below the preterminal valve represented $4.8 \%$ out of the 82 TSFL procedures. The rate of TSFL recanalizations was $4.8 \%$ below the pre-terminal valve. The Valsalva reflux flew clearly below the preterminal valve only in three cases. Therefore the percentage of cases in which the reflux reached the proximal third and midthigh went down to $3.6 \%$. All the patients were pleased with the clinical results and didn't show any visible recurrence of varicose veins.

Fifty-three patients who had crossotomy procedures were followed up with from January 2008 to December 2010. The average follow-up period was 29 months. Saphenousfemoral reflux due to neovascularization (no residual stump was detected on the femoral side of the crossotomy) was identified thanks to the Valsalva maneuver in one patient at the SFJ above the preterminal valve (this means the SFJ that remains after the section for the crossotomy procedure) and at proximal third of the thigh in two cases. The prevalence of neovascularization at 12 months was $0 \%$ and only three interventions showed neovascularization at 19 and two at 30 months; cumulative incidence $3 / 61=4.9 \%$. No varicose veins recurrence was visible and all the patients were asymptomatic.

Fifty-five patients ( 37 female, 18 male), had triple prolene ligature procedures (2004-2005). Forty-nine of them had a follow-up check one year after the operation; 21 showed recanalization with saphenous-femoral reflux; 14 were asymptomatic with moderate Valsalva reflux at the upper third of the thigh and seven were symptomatic with Valsalva reflux down to the lower leg. All recurrences occurred between the $3^{\text {rd }}$ and $6^{\text {th }}$ month after surgery. Re-operation (crossotomy) under local anaesthesia and with a minimal surgical trauma, was performed in three cases. The surgical dissection showed new vessels around the polypropylene thread. The cumulative incidence of recanalization was $21 / 56=37.5 \%$ at one year. Looking at the differences among groups (Table 1), age was not significant for neither the unilateral or the bilateral patients. In the case of the bilateral patients, the age at both interventions and that at the first intervention gave no different results. The percentage of females was higher in the crossotomy group, but it did not reach significance looking at multiple comparison. However, age and gender were further considered as factors that could affect the efficacy of this method.

The incidence rates were: 0.0017 event/ month/patient for the crossotomy group, 0.0037 event/month/patient for the TSFL group and 0.040 event/month/person for the TPL group.

Looking at survival curves (Figure 2), both the crossotomy and TSFL groups were signifi-

Table 1. Characteristics of the patients.

\begin{tabular}{lccc} 
& Crossotomy & TSFL & TPL \\
Interventions & 61 & 82 & 56 \\
Patients & $53: 45 \mathrm{U}, 8 \mathrm{~B}$ & $71: 60 \mathrm{U}, 11 \mathrm{~B}$ & $55: 54 \mathrm{U}, 1 \mathrm{~B}$ \\
\hline Gender & $6 \mathrm{M} / 47 \mathrm{~F}$ & $19 \mathrm{M} / 52 \mathrm{~F}$ & $18 \mathrm{M} / 37 \mathrm{~F}$ \\
Age, years (Bilateral=1 ${ }^{\circ}$ intervention) & $57.8(12.3)$ & $58.3(12.3)$ & $62.9(12.4)$ \\
\hline Age, years (Bilateral=both) & $58.6(12.6)$ & $58.2(12.4)$ & $63.0(12.3)$ \\
Follow-up time (months) & $29.0(18.5-34.0)$ & $14.0(12.0-20.0)$ & $12.0(6.0-12.0)$ \\
\hline
\end{tabular}

TSFL, triple saphenous flush ligation; TPL, triple polypropylene ligation; $\mathrm{U}$, unilateral; B, bilateral.

Table 2. Cox regression results, using crossotomy as control group (0).

\begin{tabular}{lcccccc} 
& B & SE & Sig. & Exp (B) & \multicolumn{2}{c}{$95 \%$ CI } \\
TSFL(1) & 1.376 & 0.835 & 0.099 & 3.960 & 0.770 & 20.356 \\
TPL(2) & 4.306 & 1.026 & $<0.001$ & 74.161 & 9.928 & 553.949 \\
\hline Age & 0.020 & 0.016 & 0.216 & 1.020 & 0.989 & 1.052 \\
Gender & -0.430 & 0.397 & 0.280 & 0.651 & 0.299 & 1.418 \\
\hline
\end{tabular}

$\mathrm{B}$, variable coefficient in the Cox regression; SE, standard error; Sig, significance; Exp(B), exponential of B. Exp(B) is the ratio of the hazards between two individuals whose values of $\mathrm{x}$ variable differ by one unit when all other covariates are held constant. It can be interpreted as a relative risk; $\mathrm{CI}$, confidence interval; TSFL, triple saphenous flush ligation; TPL, triple polypropylene ligation. 
II

crossotomy

titanium clip in red

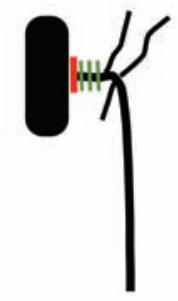

TSFL

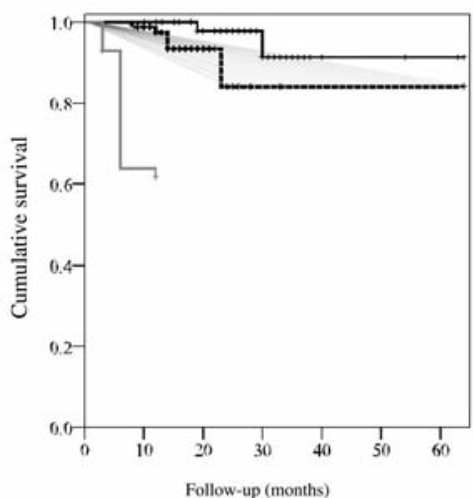

Figure 2. Survival curves of crossotomy (black continuous line), triple saphenous flush ligation (black dashed line) and triple polypropylene ligation (grey continuous line). Crosses are censored data.
Figure 1. Saphenous femoral disconnection with division on the left (crossotomy) and saphenous femoral multiple ligation without division on the right (TSFL). cantly different from the TPL group $(\mathrm{P}<0.001)$, but the difference was not significant between them $(\mathrm{P}=0.08)$ if we consider all the surgical interventions as independent. Results did not change when only unilateral interventions were considered or in the case of bilateral interventions only the first one was taken (not shown). The same model was weighted for gender and age, and the results of the Cox regression model are reported in Table 2 (crossotomy group was the control). Gender and age were not significant, and the results substantially confirmed those of the unweighted model.

\section{Discussion}

The conservative saphenous-femoral disconnection is performed, according to the CHIVA strategy, in order to preserve the drainage of the collateral veins and also to disconnect a closed shunt. The drainage of the collateral veins of the SFJ is preserved in order to prevent defects of flow in these veins, that can trigger recurrences do to neovascularization. The CHIVA strategy focuses on the treatment of the shunt escape point in order to eliminate shunt pressure. A closed shunt is a recirculation with pressure overload caused by a district skip. The reflux arises from an escape point (N1-N2), flows into superficial veins and returns into deep veins through a reentry point again reaching again the escape point.

The objective of this trial was to assess the durability of SFJ closure. This trial is not a randomized controlled trial but groups are homogeneous, there being only one surgeon and three Duplex scan operators. The survival curves show a clear difference of the crossoto- my and TSFL groups and the TPL group, but also show that the difference was not significant between them especially if we consider the cases of recurrence with reflux at the thigh during the systole of Valsava.

Contrary to TPL, TSFL didn't show significant recurrences. This suggests strongly the incidence of the technique as thread features on the recanalization.

The short recurrent Valsalva reflux after TSFL is explained by the narrow and long residual reflux lumen (three superposed ligations along 5 to $10 \mathrm{~mm}$ ) at the procedure site, which represents a resistance that hampers a massive consequent flow. In all the reflux recurrences in the crossotomy and TSFL groups, patients were asymptomatic and nonvaricose veins recurrence was visible.

However, the difference between TPL and TSFL, both techniques of SFJ disconnection without division, is evident. The analysis of TPL follow up suggests us that the polypropylene thread used, even if of size 0 , may cause a necrosis with a section of the vein wall. The formation of neovascularization around the ligations may be a consequence. This conclusion has been confirmed by the evidence we saw in the three patients that underwent re-do surgery. For this reason we have decided to use a less elastic overcoated thread of larger size in order to prevent this occurance. Despite these data, observation of a greater number of TSFL controls would be useful.

\section{Conclusions}

Crossotomy is the saphenous-femoral disconnection technique of reference in the implementation of the CHIVA strategy. Crossotomy is a refined surgical technique especially in overweight patients. The efficacy of the TSFL technique could be considered equal to that of crossotomy. The practical impact of TSFL is to provide a safe, reliable and durable mini-invasive open surgery for sapheno-femoral disconnection in outpatients, while TPL ligation is not reliable.

Saphenous-femoral recanalizations were asymptomatic in both TSFL and crossotomy groups, thanks to the draining effect of the spared saphena trunk, and consequently thanks to the quality of the re-entry point. Both crossotomy and TSFL showed no surgical complications. TSFL is easier and quicker than crossotomy because of the absence of vessel division. On the basis of what has been observed, both crossotomy and TSFL are suitable techniques for eliminating femoralsaphenous refux at the terminal valve escape point in case of incontinence of the terminal valve. Both techniques are adequate to maintaine the results of the disconnection/ligation over time, with the advantage of the conservation of the drainage of all the collateral veins of the SFJ. An ethics committee consultation has not been considered necessary, as the ligature of confluent veins has been used in clinical practice for years since the articles published by Trendelenburg ${ }^{14}$ until today. ${ }^{15}$

\section{References}

1. Zamboni P, Cisno C, Marchetti F, et al. Minimally invasive surgical management of primary venous ulcer vs. compression treatment: a randomized trial. Eur J Vasc Endovasc Surg 2003;25:313-8.

2. Zamboni P, Gianesini S, Menegatti E, et al. Great saphenous varicose vein surgery without sapheno-femoral disconnection. 
Br J Surg 2010;97:820-25.

3. Carandina S, Mari C, De Palma M, et al. Varicose vein stripping vs haemodynamic correction (CHIVA): a long term randomised trial. Eur J Vasc Endovasc Surg 2008;35:230-7.

4. Parés J0, Juan J, Tellez R, et al. Varicose vein surgery: stripping versus the CHIVA Method: a randomized controlled trial. Ann Surg 2010;251:624-31.

5. Iborra-Ortega E, Barjau-Urrea E, Vila-Coll $\mathrm{R}$, et al. Comparative study of two surgical techniques in the treatment of varicose veins of the lower extremities: results after five years of follow-up. [Estudio comparativo de dos técnicas quirúrgicas en el tratamiento de las varices de las extremidades inferiores: resultados tras cinco años de seguimiento]. Angiología 2006;58: 459-68.
6. Brake M, Lim CS, Shepherd AC, et al. Pathogenesis and etiology of recurrent varicose veins. J Vasc Surg 2013;57:860-8.

7. Delfrate R. Manuale di emodinamica venosa degli arti inferiori. Cremona: Fantigrafica Ed.; 2010.

8. Delfrate R, Grilli N. A new diagnostic approach to varicose veins: haemodynamic evaluation and treatment. Folgaria: Lorena Dioni Publ.; 2014.

9. Franceschi C. La cure hemodynamique de l'insuffisance veineuse en ambulatoire. J Malad Vasc 1997;22:91-5.

10. Franceschi C. Theorie et pratique de la cure conservatrice et hemodynamique de l'insuffisance veineuse en ambulatoire. Précy-sous-Thil: De L’Armançon ed.; 1988.

11. Franceschi C, Zamboni P. Principles of venous hemodynamics. New York: Nova Science Publ.; 2009.
12. Bellmunt-Montoya S, Escribano JM, Dilme J, Martinez-Zapata MJ. CHIVA method for the treatment of varicose veins (Protocol). Cochrane Database Syst Rev 2012;2: CD009648.

13. Delfrate R. Moderni fondamenti di diagnostica emodinamica dell'insufficienza venosa degli arti inferiori. Cremona: Ed. Fantigrafica; 2013.

14. Trendelenburg F. Ueber die Unterbindung der Vena saphena magna bei Unterschenkelvaricen. Beitrage Klin Chir 1890; 7:195-210.

15. Carroll C, Hummel S, Leaviss J, et al. Clinical effectivness and cost-efettivness of minimally invasive techniques to manage varicose veins: a systematic review and economic evaluation. Health Technol Assess 2013;17(48). 